\title{
Mechanical Properties Evaluation of Cold-Curing Acrylic Resin Reinforced by Banana Peels' Fibers.
}

\author{
Mohammed R. Gharkan ${ }^{1}$, Ahmed H. Ali ${ }^{1}$, Hossam E. Salman ${ }^{2}$, Bassam I. Khalil ${ }^{1}$ \\ Department of Materials Engineering, Branch of Polymer Materials Engineering, University of Technology, ${ }^{2}$ \\ University of Al-Esraa, Department of Dentistry \\ 130026@uotechnology.edu.iq
}

\begin{abstract}
Although low mechanical properties of Acrylic resin, it is the most popular used substance as based denture materials. This drawback could be treated through adding reinforcing phase. In this work washing and non-washing banana peels as kind of natural fibers with different weight fractions, (5, 10, 15 and 20) \% wt. and standared Acrylic, for compartion purpose, are used to prepare composite specimens by modeling method on order to study possibility of reinforcing cold-curing acrylic resin by banana peels. Peels had been dried, crashed, and washed by distillation water before using in the work. Unwashed peels also used for comparing purpose. Hardness, impact, and tendsile tests were performed to evaluate the development in mechanical properties of the prepared composite. Generally the results show that washed banana peels have more effective than that of un-washed peels, adding banana peels could improve hardness of composite and hardness increase with increasing weight fraction of peels till specific value, (10) \% wt. where highest hardness recorded, using high weight fraction of reinforcing phase could negatively effect into mechanical properties of composite, Also the results show that using banana fibers as reinforce phase, didin't improve impact strength, fracture toughness, and modules of elasticity properties of acrylic.
\end{abstract}

Keywords: Banana peels' fibers, Cold-Curing Acrylic Resin, Composites, mechanical properties

Paper History : (Received:16-5-2018 ;Accepted:11-112018)

\section{Introduction}

In the most recent decade, the utilization of composite materials has expanded extensively, because of their lightweight and elite highlights. [1]. A significant exertion has been dedicated to improve the properties and nature of the composite materials to meet designing necessities. [2]. Using natural and friend environmental materials, is the global trend recently. Natural fibers had been applied for developing composite materials in many researches, as their advantages over synthetic fibers like: low cost, easy handling during process, low density, non-toxicity, and relatively good strength to weight ratio [3]. It would be extremely interesting if natural fibers could be utilized rather than glass fibers as reinforcing phase in structural applications. The natural fiber segments might be wood, hemp, sisal, coconut thread, jute, Bamboo, abaca, banana leaf fibers, and wheat straw.

Banana fibers is a waste so, with no including cost input, banana fiber can be acquired for industrialized purposes. Indeed, banana fibers showed noticeable improvement in mechanical properties when it added as strengthening phase to polyester matrix. Generally, using natural fibers for reinforcing polymer could lower the required needed energy for production by $80 \%$, reduce the cost about $5 \%$, and decreas the weight of component by $10 \%$ comparing with using glass fibers [4,5]. However, using natural fibers has some limitation such as: degradation in moistness, poor surface bond with polymers, non-uniform fibers' length, not appropriate in elevation temperature applications, finally weakness to fungal growth and insects attacks [6]. Since improving bonding between polymeric matrix and natural fibers, is very vital factor to improve composite mechanical properties, many treatments could applied into natural fibers to remove presented impurties like lignin, wax, which reduce compability between the two phases, so improve adhesion strength like, treatment by alkaline, bleaching, acetylation and steaming. Among these treatments, chemical treatments are the most effective method to remove unwanted materials like wax, lignin, and extractives, since present of these materials could negatively affect into compatibility between polymer and natural fibers. However, applying these treatments is limited to industrial applications as high cost, and hazardous of this treatments [3]. Cold-curing PMMA, sometimes called self-curing acrylic, is auto-polymerized. That means the reaction of polymerization starts immediately when powder and liquid components have mixed together. Where initiator, benzoyl peroxide, which originally present in PMMA spheres has been activated by chemicals, so in this instance no heat necessary to complete polymerization reaction. Dimethyl-ptoluidine, a tertiary amine, is usually used for activating the polymerization reaction. When polymerization has begun, the reaction has the same procedure of heat cured reaction [7]. Generally, Acrylic has poor mechanical characteristic However, its mechanical properties can be developed by adding reinforcing phase to the acrylic matrix [8]. In this work, waste banana peels, washed and unwashed, with different weight fractions were used on order to improve mechanical properties of cold-curing acrylic resin.

\section{Materials and methods}


2.1 Materials: following materials had been used to perform the work:

1. Self-curing acrylic resin, produced by Duracryl ${ }^{\circledR}$ plus, which consisting from two constituents, powder part and liquid part: Powder part is : PMMA copolymer (fine particle size), (1-2) \% initiator (Di-benzoyl peroxide DBP), and (1) $\%$ Pigment.

Liquid part is : Methyl methacrylate monomers MMA, (12) $\%$ cross-linking agents (Glycol di-methacrylate), $(<1) \%$ inhibitor (Hydroquinone), and activator (Dimethyl-ptoluidine).

2. Banana peels: Banana peels had been brought from the banana fruit. The peels had been separated, dried at $(50)^{\circ} \mathrm{C}$ for (48) hr. then crushed, and finally sieved to get fine fibers that passed through a 50 mesh before washing.

The banana peels' had been washed by distilled water, (50) gm of crushed peels to (500) $\mathrm{ml}$ water for (10) min. at room temperature. Once the washing had completed, the solution had been filtered by a vacuum filter, then the fibers had been dried at (50) ${ }^{\circ} \mathrm{C}$ for (48) hr. by oven.

\subsection{Specimens' preparation procedure:}

Resin specimens were equipped by adding cross-linked liquid part to powder part with (3:1) ratio. Composite specimens were prepared by mixing the three ingredient together, powder part, cross-linked liquid and banana peels, before pouring into molds. Three different shapes and dimensions molds were used, according to the specification of tests: hardness, impact, and tension. Vaseline was used to grease molds for facilitating specimens' separation from mold after solidification. Finally, different weight fractions of washed and unwashed fibers were depended, $(5 \%, 10 \%, 15 \%$, and $20 \%)$, to perform the research.

\section{3 mechanical tests:}

\subsubsection{Hardness test:}

Hardness tests were performed by using Shore hardness type (D) instrument, according to specification ASTM (D2240) [9]. The tests were performing at room temperature and the average hardness of value of many tests was depended. Hardness specimens and shore hardness instrument are shown in Figure 1.

\subsubsection{Impact test:}

Impact test is a measure of material strength under high rate of loading (sudden loading). Impact strength is calculated from the following equation:

$G_{c}=\frac{U_{c}}{A}$

Where:
Gc: is a material's toughness $\left(\mathrm{J}^{-\mathrm{m}^{-2}}\right), \mathrm{Uc}$ : is the impact energy $(\mathrm{J})$, and A: is a specimen cross section area $\left(\mathrm{m}^{2}\right)$ [10].

Fracture toughness describes the capability of a material, which containing a crack, to resist fracturing, expressed by relation:-

$\boldsymbol{K}_{\boldsymbol{c}}=\sqrt{\mathbf{G}_{\mathbf{c}} \mathbf{E}}$

Where:

$\mathrm{Kc}$ : is the fracture toughness of material $\left(\mathrm{MPa} \cdot \mathrm{m}^{1 / 2}\right), \mathrm{Gc}$ : is the impact strength of material $\left(\mathrm{J}^{\mathrm{m}} \mathrm{m}^{-2}\right)$, and $\mathrm{E}$ : is elastic modulus of material (MPa) [10].

Izod impact test model, is applied for testing composites specimens. These tests were performed at room temperature according to specification (ISO- 179) [10]. Figure 2 shows a schematic design of specimen, used for impact test, and Figure 3 shows the specimens after performing the test and impact instrument. Impact strength and fracture toughness were calculated from previous mentioned relations

\subsubsection{Tensile test:}

The tensile tests were performed according to ASTM specification (D638M) at room temperature [11]. The test involves an axial tensile load being applied to a standard tensile specimen of a rectangular cross section, with a constant strain rate at about (5) $\mathrm{mm} / \mathrm{min}$. Figure 4 shows the schematic with dimensions of a tensile test standard specimen, Figure 5 shows prepared specimens after performing tests for variant reinforcing weight fraction specimens and tensile test instrument.

\section{Results and Discussion:}

The effect of reinforcing cold-curing acrylic resin, by banana peels, in mechanical properties: hardness, impact strength and tensile strength, had been discussed according to results of performed tests, which plotted as a function to weight fractions percentage of reinforcing phase, for washed W.P. and unwashed Un-W.P. banana peels as following.

\subsection{Hardness test:}

Table 1 shows the results of average hardness value to the cold curing acrylic resin reinforced with different weight fractions of washed and unwashed banana peels, Figure 6 displays the relation between the hardness and the weight fractions of the washed and unwashed banana peels.

According to the figure, hardness increases with increasing weight fraction of banana peels. In both cases, washed and unwashed peels, till spesific value, where maximum hardness value was recorded, (10) \% wt. of peels. This increasing may relate to restriction of resin chain movement by natural fibers of peels, so increasing material resistance to pentration. However, it can notice 
that reinforcing by washing peels give higher hardness vales than that of unwashed peels. The explain of this facts is washing peels by water process can help removing impurities materials that associate with natural fibres, which in charge of reduction of reinforcing efficiency, either because it has lower mechnical properties than fibers or by reduction bonding strength between two phases becous it has lower compability with polyer matrix than natural fibers. Adding high peels weight fractions, more than (10) \% wt., could produce less strengthening efficiency, since failure of matrix to surround all amount of added fibres perfectly, consequently creation voids and discontinouse points between fibers and matrix which has deterioration effects into mechanical properties of composite, or may be cration agglomeration of peels site within matrix which inversely into mechanical properties of composite as absent bonding betwwen peels and matrix in these sites.

\subsection{Impact Test:}

As mentioned impact tests were performed according to IZO model. Tables 2,3 show the results data of impact energy, and corresponding impact strenght and fracture toughness, acoording to equations $(1,2)$, of composite specimens in both cases, washed peels W.P. and unwashed peels Un-W.P., Figures 7-9 show the effect of peels weight fraction into mentioned charactrstics.

Toughness is ability of materials to withstand applied energy upto fracture, usually tough materials are those which have high strength and ductility, ductile materials has higher toughness than brittle materails even if the last have higher yeild stress and tensile strength values[12].

According to the recorded results in tables (2,3), impact energy, impact strength, and fracture toughness of tested composite specimens are lower than that of based acrylic resin specimen. The reason of this reduction may be related to convert mechanical behavior of composite structural to be more brittleness with increasing banana peels weight fraction, since brittle nature charcteristic of peels comparing with acrylic resine. It can be noticed that increasing reinforcing weight fraction of banana peels could reduce toughness of composite since increasing brittle material percentage at the expense of resine, or may be related to increasing voides and defects points in matrix as non perfact bonding between the two phases with increasing peels fraction. Also it can be noticed according figures 7-9, composite with washed peels were more toughness than that of unwashed peels as washing process can reduce the present of impurties that associated natural fibers in peels, where as mentioned previously the effect of these impurities can decrease mechanical toughness properties of peels and reducing bonding strength between two phases [13].

\subsection{Tensile Test:}

Tensile test involves an axial tensile load being applied to a standard tensile specimen of a rectalar cross section with a constant strain rate at about (5) $\mathrm{mm} / \mathrm{min}$. the initial part of the stress-strain curve is linear where the specimen behaves in an elastic manner. Beyond the limit of proportionality, which refers as a yield point. After the yielding of material, the stress- strain curve deviates due to the deformation of specimen in plastic manner. An increasing in the plastic deformation continued with matching increasing stress, till the fracture occurred in the specimen. Table 4 shows the varying in: maximuim tensile strength, maximuim strain, and elastic modules values with varying of peels weight fraction, and Figures 10-12 show relation between: elastic modolus, maximuim tensile strength, and maximum strain and peels weight fraction.

Basically mechnical properties of composite is higly depending upon mechanical properties of its consitituents materials and bonding strength between consitituents phases. Breaking or separating of fibers from matrix resin under applied load can accelerate failure of composite drametically, since matrix don't transfer loads to fibers any more, so fibers act as holes, and matrix will stand whole applied load, that leading to increase locally stresses without any additional load, consequently increasing of strain till fracture occure.

According to figure 10, It can be noticed decreasing modoluse of elasticity with increasing peels weight fraction. in both cases, (washed peels and un-washed peels). The explain of this behavior may be related to low modolus of elasticity of peels comparing with that of acrylic matrix, or inversly effects of high weight fraction of peels in properties as previously mentioned. Maximum tensil strength chart, figure 11 , shows simple increasing in strength when adding (5) \% wt. of washed peels while decreasing of strength for same percentage for un-washed peels, the explain may be related to effect of impurties which associated with peels, as previously mentioned. Then tensile strength values had deterioration with increasing peels weight fraction, the explain to this behavior may be related same resaons which earlier mentioned in hardness test discussion. Finally maximum strain chart, figure 12, shows incresing in strain value when added (5) \% wt. of peels (in both cases) following by deterioration in values with increasing peels weight fraction. The explain to this behavor may be related to negative effect of high weght fraction of peels which lead to accelrate failure and fracture occurrence, addition to brittle nature of natural fibers comparing with Acrylic resin. However, this results agree with many previous researches which used natural fibers as reinforcing phase [14].

\section{Conclusions:}

1. Using banana peels to reinforce cold curing acrylic, didn't show specific improvent in mechanical properties 
2. Washing banana peels could increase strengthening efficiency.

3. Using (10) \% wt. of banana peels could improve acrylic resin hardness about $10 \%$

\section{Resources}

1. Razak, Adnan A. Abdul. "MODELING OF THE CURE OF EPOXY BASED COMPOSITE, HEATED AT CONSTANT TEMPERATURE IN CYLINDERICAL MOULD." Diyala Journal of Engineering Sciences 4.1 (2011): 1-11.

2. Shnean, Zanaib Y. "Mechanical and physical properties of high density polyethylene filled with carbon black and titanium dioxide." Diyala Journal of Engineering Sciences 5.1 (2012): 147-159.

3. Pereira, Paulo Henrique Fernandes, Kelly cristina C. carvalho Benini, Cintia Yumi Watashi, Herman Jacobus Cornelis Voorwald, and Maria Odila Hilario Cioffi, characterization of high density polyethylene (HDPE) reinforced with banana peel fibers, BioResources (2013): 2351-2365.

4. Sakthivel, M., and S. Ramesh, mechanical properties of natural fiber (banana, coir, sisal) polymer composites, science park 1.1 (2013): 1-6.

5. Sapuan, S. M., M. Harimiand, and M. A. Maleque, mechanical properties of epoxy/coconut shell filler particle composites, Arabian Journal for Science and Engineering 28.2 (2003): 171-182.
6. Belmares, Hector, Arnoldo Barrera, and Margarita Monjaras, New composite materials from natural hard fibers. 2. Fatigue studies and a novel fatigue degradation model, Industrial \& engineering chemistry product research and development 22.4 (1983): 643-652.

7. Grant, Alan, Problems with polymers in dentistry, Polymer International 10.4 (1978): 241-244.

8. Vipul Asopa, S. Suresh, Meenakshi Khandelwal, Vivek Sharma, Shivalika S. Asopa, Laxman Singh Kaira, A comparative evaluation of properties of zirconia reinforced high impact acrylic resin with that of high impact acrylic resin, The Saudi Journal for Dental Research 6.2 (2015): 146-151.

9. Hayder Raheem Kareem, Study The Effect of Nano carbon Black Particle (N220) on The Properties of Polymer Composite Material, Thesis Master, 2013.

10. Rabab Asim Abdul-Aziz. Preparation and Characterization of Polymer- Ceramic Composite Biomaterial. Thesis Master, (2009).

11. American Standard and Testing Methods (ASTM)," Standard Test Method for Tensile Properties of plastic D638M-87b", Annual book of ASTM standard, Vol.(09.01), (1988).

12. CALLISTER JR, William D. Materials Science and Engineering, John willey \& Sons. Inc, New York, 2007.

13. M. S. Sreekanth, Shashank Mhaske, Prakash Mahanwar ,Effect of concentration of mica on properties of polyester thermoplastic elastomer composites, Journal of Minerals and Materials Characterization and Engineering 8.04 (2009): 271.

14. Venkateshwaran, N., and A. Elayaperumal, Banana fiber reinforced polymer composites-a review, Journal of Reinforced Plastics and Composites 29.15 (2010): 23872396.

Table 1: The results of the hardness tests of acrylic-banana peels composite

\begin{tabular}{|c|c|c|}
\hline Weight fraction & $\begin{array}{c}\text { Average hardness } \\
\text { w. p. }\end{array}$ & $\begin{array}{c}\text { Average hardness } \\
\text { un-w.p. }\end{array}$ \\
\hline $0 \%$ & 77.3 & 77.3 \\
\hline $5 \%$ & 81 & 79.3 \\
\hline $10 \%$ & 85 & 80.0 \\
\hline $15 \%$ & 65 & 66.6 \\
\hline $20 \%$ & 75 & 79.0 \\
\hline
\end{tabular}

\begin{tabular}{|c|c|c|c|c|}
\hline \multicolumn{5}{|c|}{ Table 2: Impact tests results of the prepared composite } \\
\hline \multirow{3}{*}{ Volume fraction } & \multicolumn{2}{|c|}{$\begin{array}{c}\text { Cross-sectional area, } \\
(\mathrm{A}),\left(\mathrm{m}^{2}\right)\end{array}$} & \multicolumn{2}{c|}{ Impact Energy, (Uc), (J) } \\
\cline { 2 - 5 } & w.p. & Un-w.p & w.p. & Un-w.p. \\
\hline $0 \%$ & 0.082 & 0.082 & 0.65 & 0.65 \\
\hline $5 \%$ & 0.071 & 0.068 & 0.45 & 0.37 \\
\hline $10 \%$ & 0.07 & 0.07 & 0.55 & 0.34 \\
\hline $15 \%$ & 0.076 & 0.068 & 0.50 & 0.35 \\
\hline $20 \%$ & 0.068 & 0.065 & 0.48 & 0.29 \\
\hline
\end{tabular}




\begin{tabular}{|c|c|c|c|c|}
\hline \multicolumn{5}{|c|}{ Table 3: Impact tests results of the prepared composite } \\
\hline \multirow{2}{*}{ Volume fraction } & \multicolumn{2}{|c|}{$\begin{array}{c}\text { Impact strength, (Gc), } \\
\left(\mathrm{J} / \mathrm{m}^{2}\right)\end{array}$} & \multicolumn{2}{c|}{$\begin{array}{c}\text { Fracture toughness, (Kc), } \\
(\text { MPa. m) }\end{array}$} \\
\cline { 2 - 5 } & w.p & Un-w.p. & w.p. & Un-w.p. \\
\hline $0 \%$ & 7.92 & 7.92 & 6.71 & 6.71 \\
\hline $5 \%$ & 6.33 & 5.44 & 5.21 & 5.18 \\
\hline $10 \%$ & 7.85 & 4.85 & 6.26 & 5.84 \\
\hline $15 \%$ & 6.57 & 5.14 & 5.37 & 4.7 \\
\hline $20 \%$ & 7.05 & 4.46 & 5.24 & 5.29 \\
\hline
\end{tabular}

Table 4: Maximum tensil strength, Maximum strain, and Young modules of acrylicbanana peels composite

\begin{tabular}{|c|c|c|c|c|c|c|}
\hline \multirow{2}{*}{$\begin{array}{c}\text { Weight } \\
\text { fraction }\end{array}$} & \multicolumn{3}{|c|}{ Washed peels } & \multicolumn{3}{c|}{ Unwashed peels } \\
\cline { 2 - 7 } & $\begin{array}{c}\text { Max. } \\
\text { Stress } \\
\text { M.Pa }\end{array}$ & $\begin{array}{c}\text { Max. } \\
\text { Strain }\end{array}$ & $\begin{array}{c}\text { Modoluse } \\
\text { of elasticity } \\
\text { G.Pa }\end{array}$ & $\begin{array}{c}\text { Max. } \\
\text { Stress } \\
\text { M.Pa }\end{array}$ & $\begin{array}{c}\text { Max. } \\
\text { Strain }\end{array}$ & $\begin{array}{c}\text { Modoluse of } \\
\text { elasticity } \\
\text { G.Pa }\end{array}$ \\
\hline $0 \%$ & 30 & 5.25 & 5.714 & 30 & 5.25 & 5.7 \\
\hline $5 \%$ & 31.5 & 7.4 & 4.3 & 28 & 6.5 & 4.25 \\
\hline $10 \%$ & 27 & 6.2 & 5.0 & 29 & 5.8 & 4.35 \\
\hline $15 \%$ & 16.2 & 4.8 & 4.4 & 22 & 5.0 & 3.37 \\
\hline $20 \%$ & 30 & 5.25 & 3.9 & 19 & 4.8 & 3.98 \\
\hline
\end{tabular}

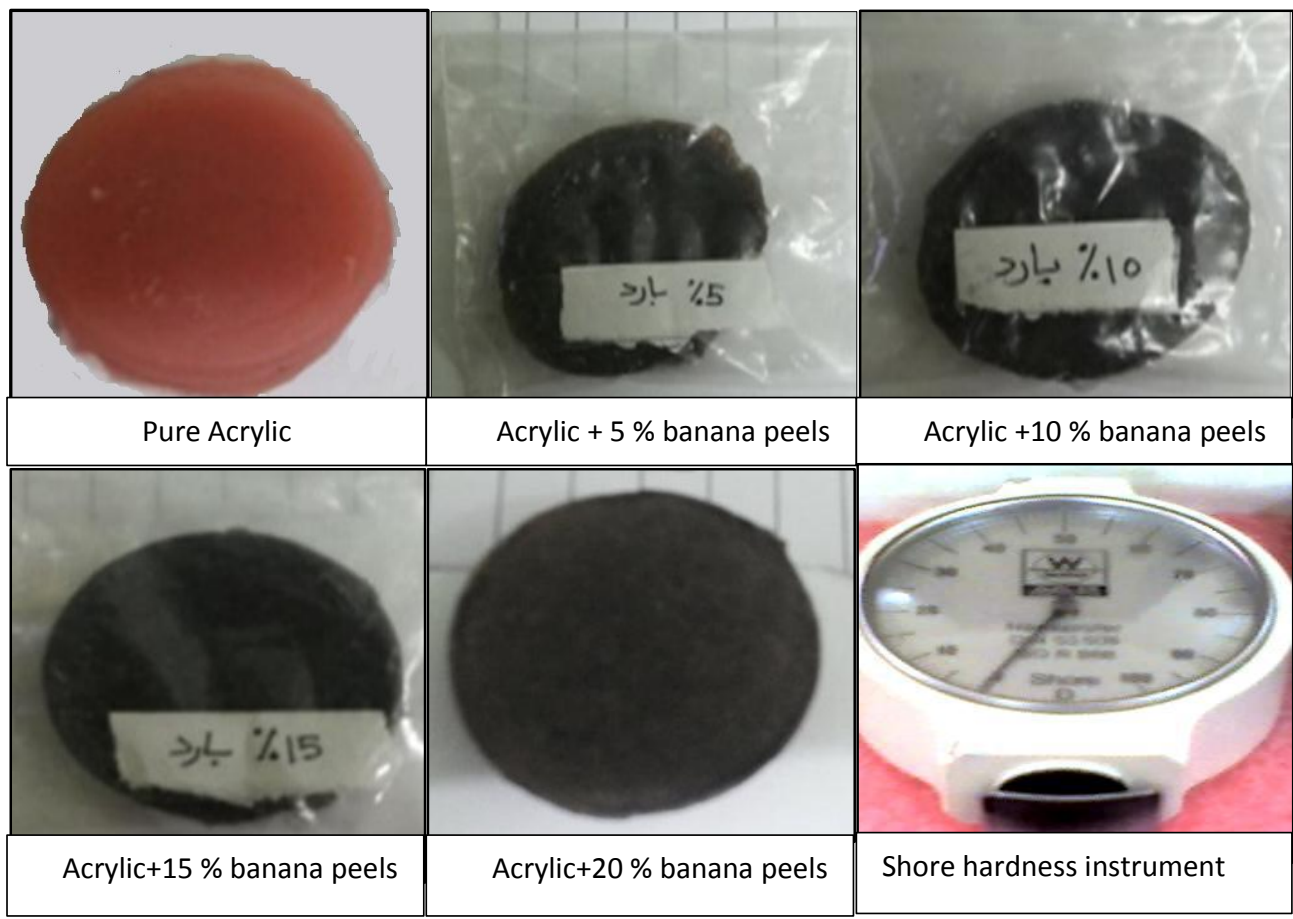


Figure 1: Hardness specimens and shore hardness instrument

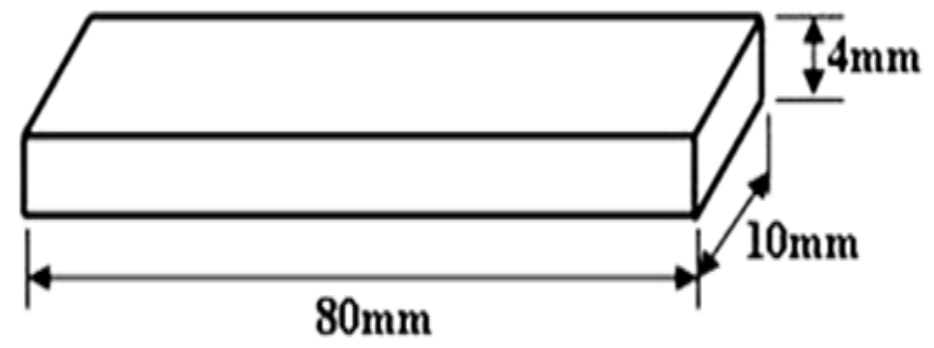

Figure 2: Schematic design of impact test specimen [10]

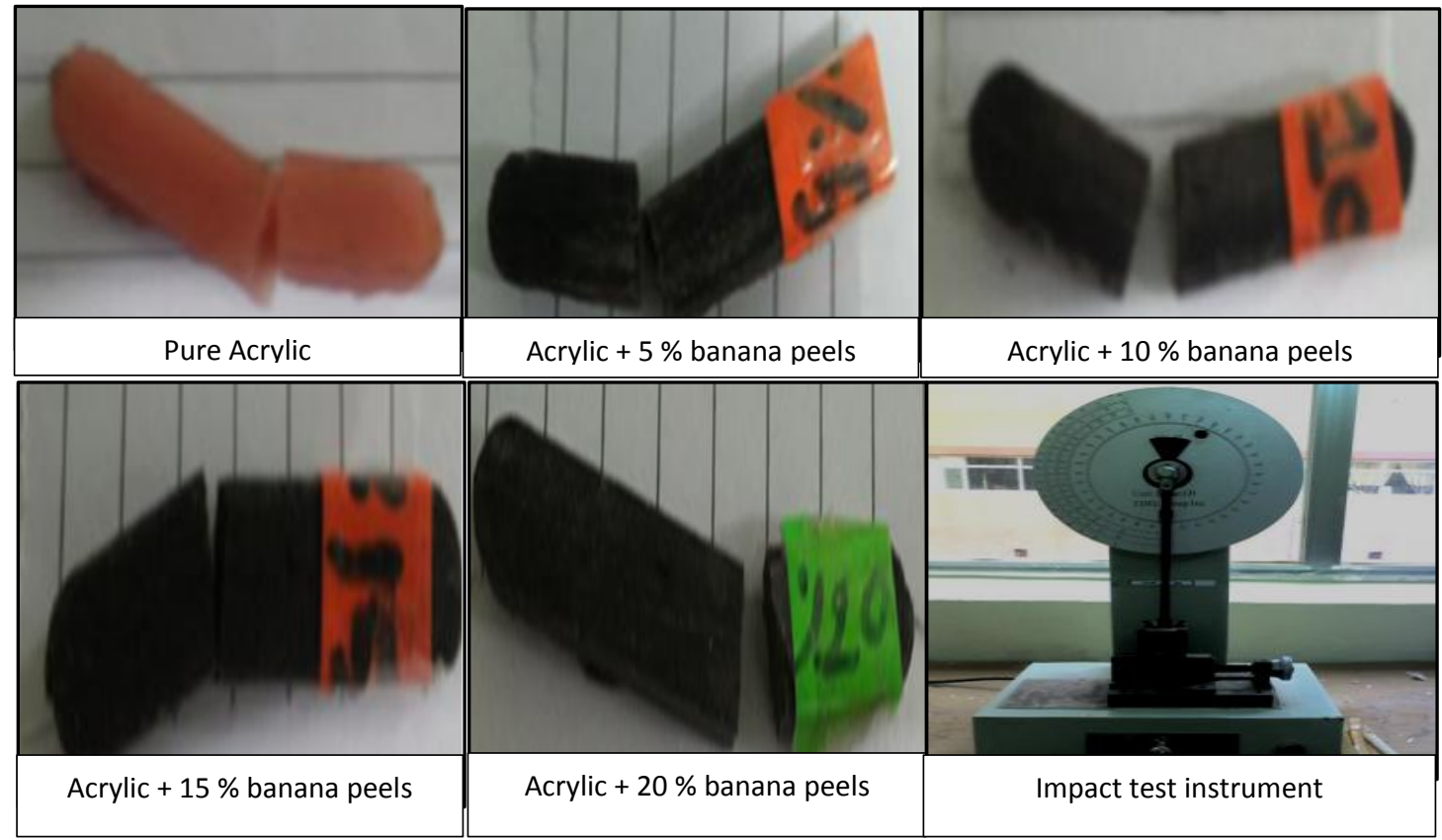

Figure 3: the specimens after performing the test and impact instrument

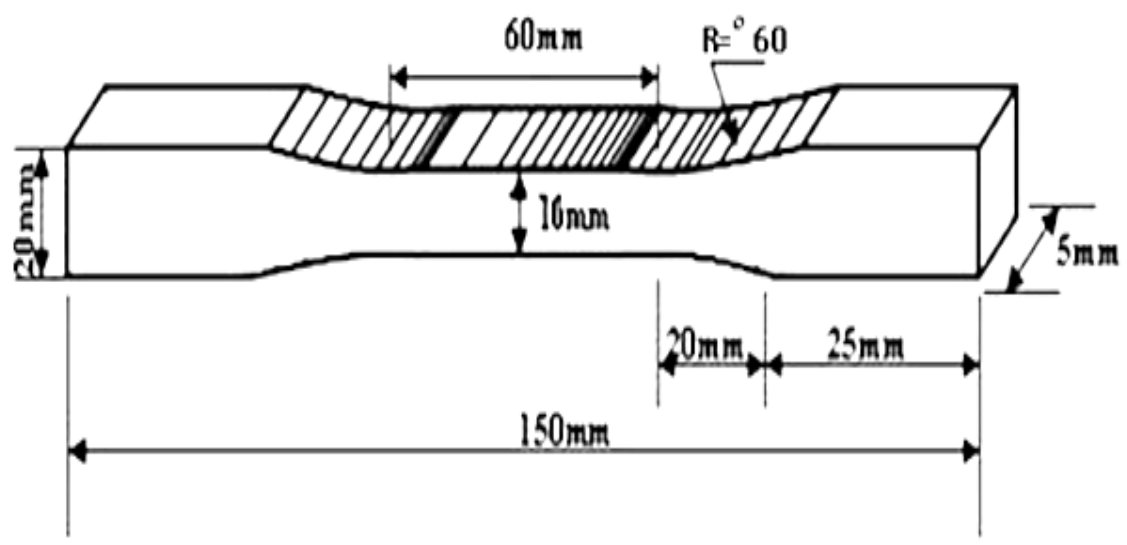

Figure 4: Schematic of tensile test specimen [11] 

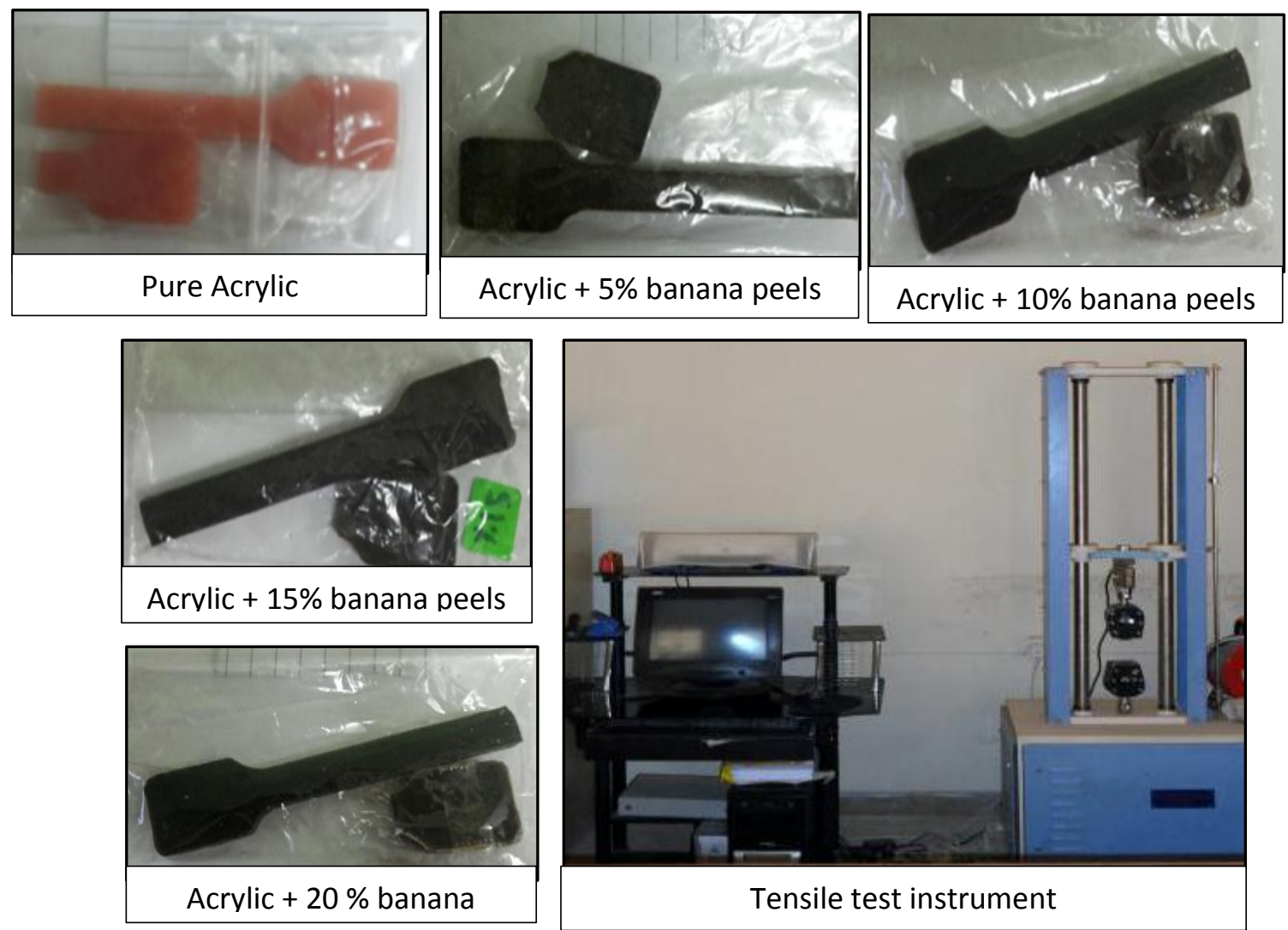

Figure 5: Tnsile test specimens after performing test $\&$ tensile instrument

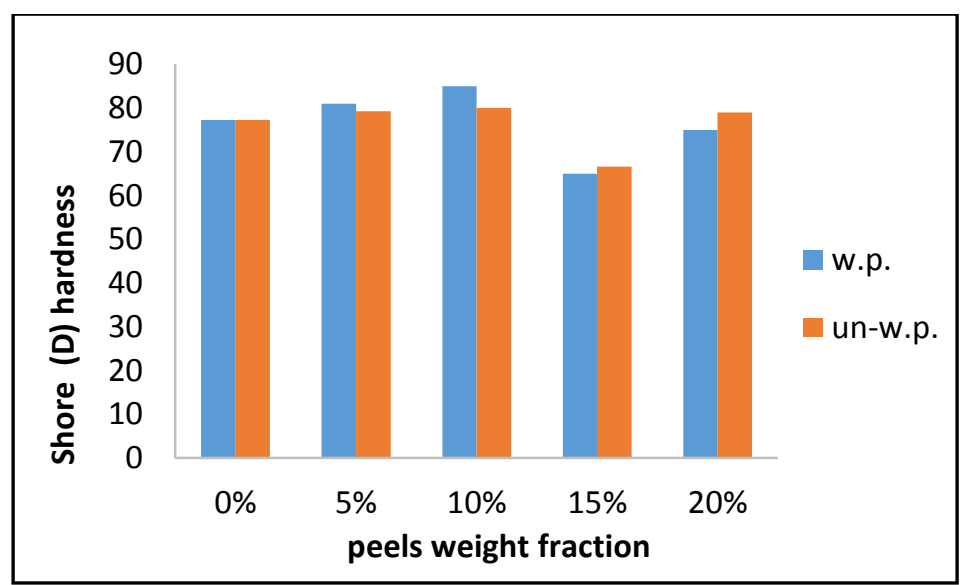

Figure 6: The relation between hardness and the weight fraction of the washed and unwashed peels 


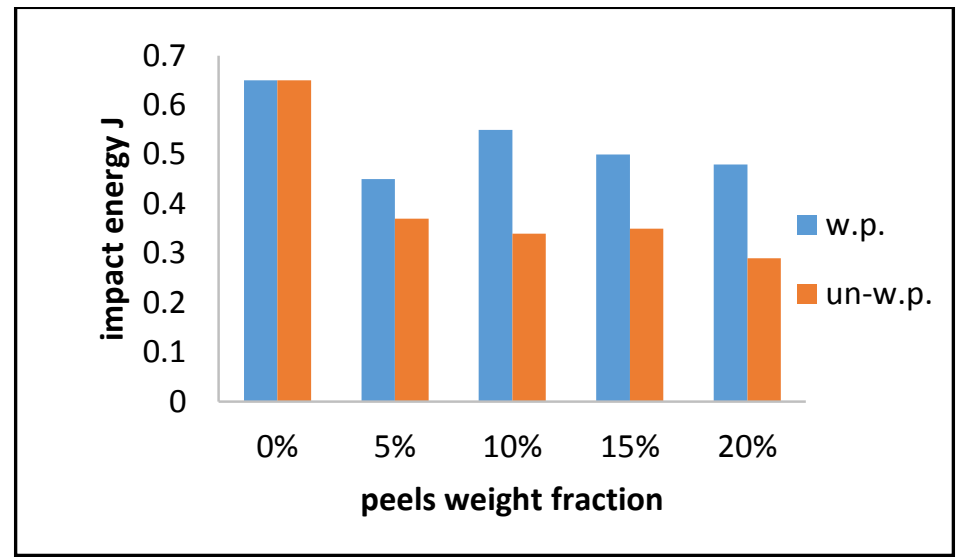

Figure 7: The relation between the impact energy and the weight fraction of washed and unwashed banana peel

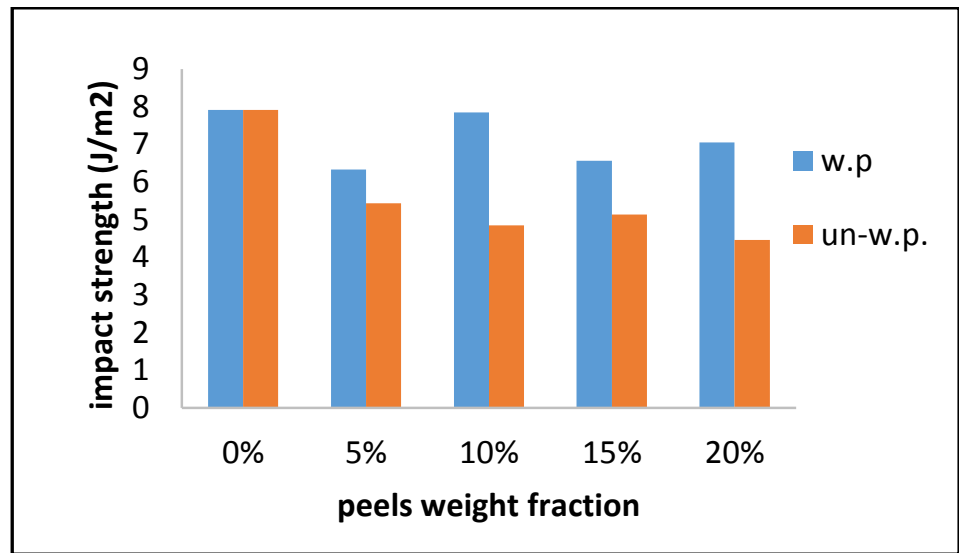

Figure 8: The relationship between the impact strength and the weight fraction of washed and unwashed banana peels

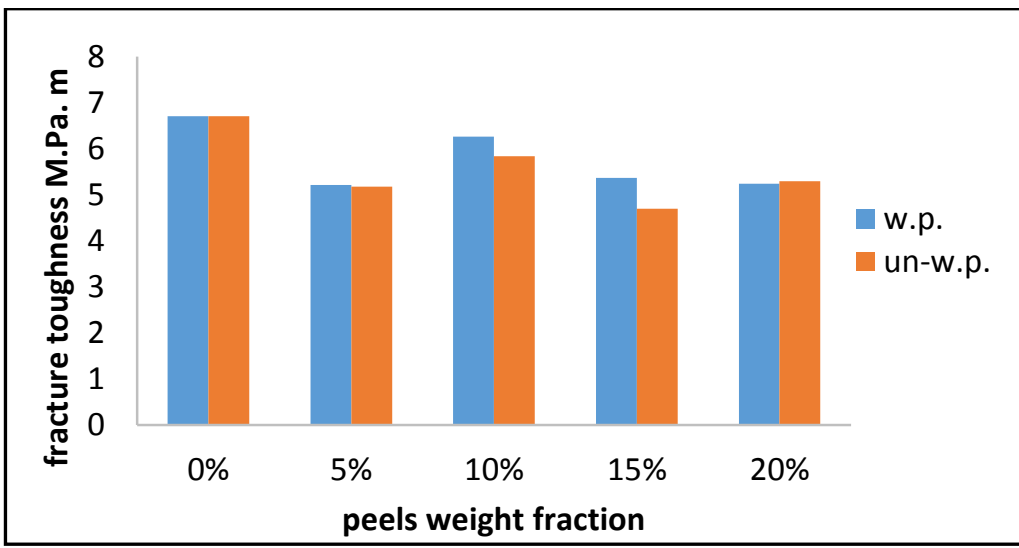

Figure 9: The relation between weight fraction and fracture toughness 


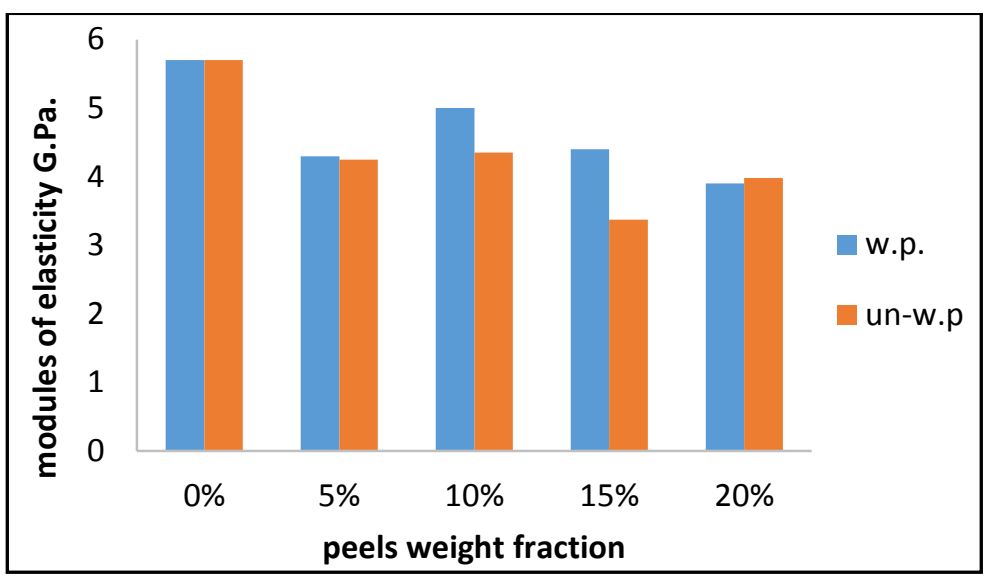

Figure 10: The effect of peels weight fraction into modules of elasticity

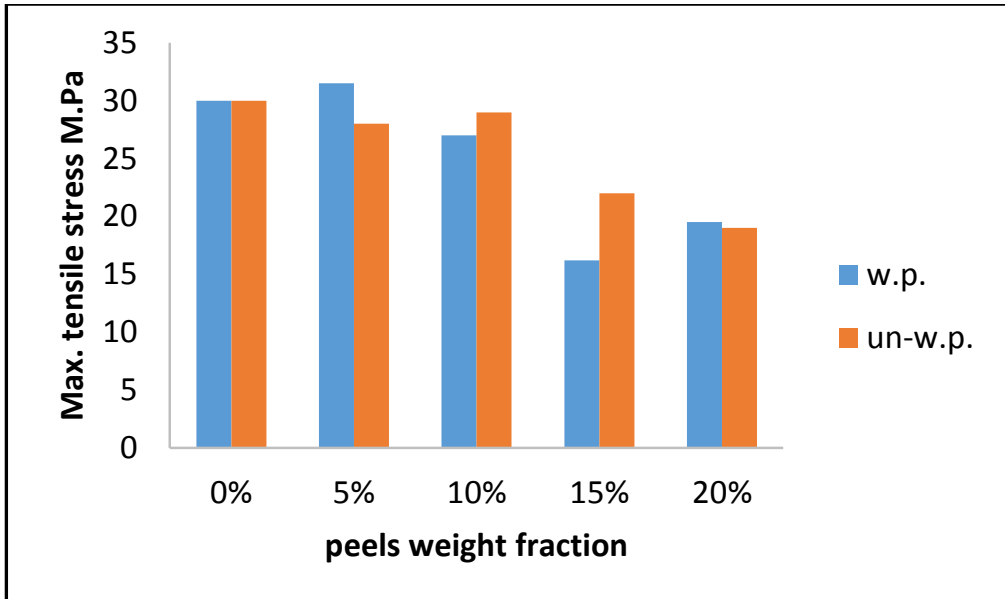

Figure 11: The effect of peels weight fraction into maximuim tensile strength

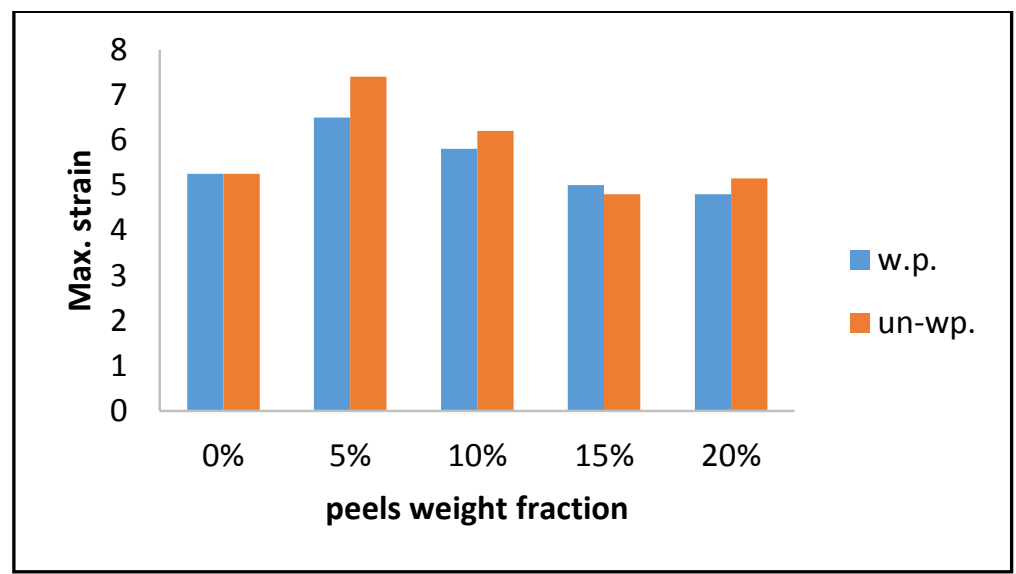

Figure 12: The effect of peels weight fraction into maximuim strain 